Homology, Homotopy and Applications, vol.7(2), 2005, pp.95-108

\title{
HPT AND COCYCLIC OPERATIONS
}

\section{ROCIO GONZALEZ-DIAZ AND PEDRO REAL}

\author{
(communicated by James Stasheff)
}

\begin{abstract}
We reinterpret the classical theory of cocyclic operations in terms of permutations and homotopy equivalences of explicit chains. The essential tools we use are Homological Perturbation Theory and Eilenberg-Zilber Theorem. The main objective of this technique is the final identification of cohomology operations at cochain level.
\end{abstract}

\section{Introduction}

L. Kristensen [18] initiated the study of the relationship between cohomology operations and simplicial cochain operations. In $[\mathbf{1 7}, \mathbf{1 8}]$, a representation result for stable primary and secondary cohomology operations in terms of cochain maps is given and some results for the evaluation of secondary and tertiary operations in low dimensions are obtained. Klaus [16], using representability results and the cohomology of Eilenberg-MacLane spaces, extended Kristensen's results to prove that any cohomology operation mod $m$ (or more precisely, cocyclic operations) can be described in terms of polynomials of coface operators at the cochain level.

In this paper, cocyclic operations are considered from the algorithmic perspective given by Homological Perturbation Theory (HPT) $[\mathbf{8}, \mathbf{1 5}]$. The fundamental data of HPT consists of a special chain homotopy equivalence called a contraction $[\mathbf{2 0}]$. More concretely, we design a method for obtaining explicit formulae for cocyclic operations as follows. First, we consider a cycle representative of a homology generator of a subgroup of the symmetric group $\mathcal{S}_{p}$. Next, we apply a perturbation process to obtain a first approximation of the formulae in terms of permutations and the component morphisms of a given Eilenberg-Zilber contraction [6]. As an example, we apply this technique to the particular case of Steenrod and Adem cohomology operations $[\mathbf{2 4}, \mathbf{2 5}, \mathbf{1}, \mathbf{2}]$.

The paper is organized as follows. In Section 2 we introduce the theoretical background from Algebraic Topology on the concepts we use here. In Section 3, we give a procedure for computing cocyclic operations using Homology Perturbation Theory. In Section 4, we apply our method to the particular case of Steenrod and Adem cocyclic operations. Finally, Section 5 is devoted to conclusions and remarks.

Partially supported by the PAICYT research project FQM-296 from Junta de Andalucía. Computational Topology and Applied Math group, http://www.us.es/gtocoma.

Received December 11, 2003, revised July 12, 2004; published on April 22, 2005.

2000 Mathematics Subject Classification: 55S10, 05E99.

Key words and phrases: Cohomology, cocyclic operations, Homological Perturbation Theory. (C) 2005, Rocio Gonzalez-Diaz and Pedro Real. Permission to copy for private use granted. 


\section{Background}

We introduce the basic notation and terminology that we use throughout the remainder of this paper. The reference for the material in this section is [20].

Let $R$ be a commutative ring with identity $1 \neq 0$. A chain complex is a graded $R$-module $C_{*}=\oplus_{n \in \mathbf{Z}} C_{n}$ together with an $R$-module endomorphism

$$
d=\sum_{n \in \mathbf{Z}} d_{n}: C_{n} \rightarrow C_{n-1}
$$

such that $d d=d^{2}$ is zero. The map $d$ is called the differential of $C_{*}$. The kernel of $d_{n}$ is the module of $n$-cycles in $C_{*}$; The image of $d_{n+1}$ is the module of $n$-boundaries in $C_{*}$; the quotient

$$
H_{n}\left(C_{*}\right)=\operatorname{Ker} d_{n} / \operatorname{Im} d_{n+1}
$$

is the $n^{\text {th }}$ homology module of $C_{*}$. The homology class of a cycle $a$ is denoted by $[a]$. The $n^{t h}$ homology of $C_{*}$ with coefficients in a commutative ring $G$ is defined by

$$
H_{n}\left(C_{*} ; G\right)=H_{n}\left(C_{*} \otimes G\right) .
$$

Whenever two graded objects $x$ and $y$ of degree $p$ and $q$ are interchanged we apply the Koszul's convention and introduce the sign $(-1)^{p q}$. The tensor product of chain complexes $C_{*}$ and $D_{*}$ is the chain complex $C_{*} \otimes D_{*}$ with differential

$$
d_{C_{*} \otimes D_{*}}=d_{C_{*}} \otimes 1+1 \otimes d_{D_{*}} .
$$

Let $C_{*}$ be a chain complex and $G$ an $R$-module. Form the abelian group

$$
C^{n}=\operatorname{Hom}_{R}\left(C_{n}, G\right),
$$

for all $n$; its elements are the module homomorphisms $c: C_{n} \rightarrow G$, called n-cochains of $C^{*}$. The differential $d: C_{*} \rightarrow C_{*}$ induces the codifferential $\delta: C^{*} \rightarrow C^{*}$ defined by

$$
\delta^{n}(c)=(-1)^{n+1} c d_{n+1},
$$

for all $c \in C^{n}$ and for all $n$. The cohomology of $C_{*}$ is the family of abelian groups

$$
H^{n}\left(C_{*}, G\right)=\operatorname{Ker} \delta^{n} / \operatorname{Im} \delta^{n-1} .
$$

An element of $\operatorname{Im} \delta^{n-1}$ is called an $n$-coboundary and an element of Ker $\delta^{n}$ an $n$-cocycle.

A natural transformation on the family $\mathcal{C}^{*}$ of all cochains complexes is an operator $\mathcal{T}$ that commutes the following diagram for all $C^{*}, D^{*} \in \mathcal{C}^{*}$ and every cochain map $f: C^{*} \rightarrow D^{*}:$

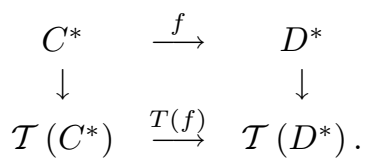

A cocyclic operation of degree $i$, is a natural transformation $O$ on $\mathcal{C}^{*}$ of the form

$$
O: C^{*} \rightarrow C^{*+i}
$$

that preserves cocycles. 
A differential graded module (DG-module for short) $M$ is a chain complex such that $M_{n}=0$ for all $n<0$. A $D G A$-module $(M, \xi, \eta)$ (we will write it simply $M$ when no confusion can arise) is a DG-module $M$ endowed with two morphisms called the augmentation $\xi: M_{0} \rightarrow R$ and the coaugmentation, $\eta: R \rightarrow M_{0}$. It is required that $\xi \eta=1_{R}$ and $\xi d=0$. A $D G A$-module morphism, $f: M \rightarrow N$, is a graded module morphism that commutes with the differentials of $M$ and $N$,

$$
f d_{M}=d_{N} f, \quad \xi_{N} f=\xi_{M} \quad \text { and } \quad f \eta_{M}=\eta_{N} .
$$

A $D G A$-algebra $(A, \mu)$ (resp. $D G A$-coalgebra $(B, \nabla))$ is a DGA-module endowed with a morphism $\mu: A \otimes A \rightarrow A$, called product on $A$, such that

$$
\mu\left(\mu \otimes 1_{A}\right)=\mu\left(1_{A} \otimes \mu\right) \quad \text { and } \quad \mu\left(\eta_{A} \otimes 1_{A}\right)=1_{A}=\mu\left(1_{A} \otimes \eta_{A}\right)
$$

(resp. $\nabla: B \rightarrow B \otimes B$, called coproduct on $B$, where

$$
\left.\left(\nabla \otimes 1_{B}\right) \nabla=\left(1_{B} \otimes \nabla\right) \nabla \quad \text { and } \quad\left(\eta_{B} \otimes 1_{B}\right) \nabla=1_{B}=\left(1_{B} \otimes \eta_{B}\right) \nabla\right) .
$$

The free $R$-algebra generated by a group $\mathcal{G}$ is a DGA-algebra denoted by $R_{*}[\mathcal{G}]$. It satisfies that

- It is zero in each degree except for degree zero, where,

$$
R_{0}[\mathcal{G}]=\left\{\sum_{a \in A} \lambda_{a} a: \quad \lambda_{a} \in R \text { and } A \text { is a finite subset of } \mathcal{G}\right\} .
$$

- The product $\mu_{\mathcal{G}}$, the augmentation $\xi_{\mathcal{G}}$ and the coaugmentation $\eta_{\mathcal{G}}$ are given by

$$
\begin{aligned}
& \mu_{\mathcal{G}}\left(\left(\sum \lambda_{a} a\right) \otimes\left(\sum \lambda_{a^{\prime}} a^{\prime}\right)\right)=\sum \lambda_{a} \lambda_{a^{\prime}}\left(a+a^{\prime}\right), \\
& \xi_{\mathcal{G}}\left(\sum \lambda_{a} a\right)=\sum \lambda_{a} \quad \text { and } \quad \eta_{\mathcal{G}}(\lambda)=\lambda \overline{0},
\end{aligned}
$$

where $a, a^{\prime} \in \mathcal{G}$ and $\lambda_{a}, \lambda_{a^{\prime}}, \lambda \in R$.

As a graded module, the reduced bar construction $\bar{B}_{*}(\mathcal{G})$ of the DGA-algebra $R_{*}[\mathcal{G}]$ is defined by

$$
\bar{B}_{0}(\mathcal{G})=R \quad \text { and } \quad \bar{B}_{n}(\mathcal{G})=\left(\operatorname{Ker} \xi_{\mathcal{G}}\right)^{\otimes n}, \quad n>0 .
$$

The element of $\bar{B}_{0}(\mathcal{G})$ corresponding to the identity in $R$ is denoted by [ ] and an element $a_{1} \otimes \cdots \otimes a_{n}$ of $\bar{B}_{n}(\mathcal{G})$ is denoted by $\left[a_{1}|\cdots| a_{n}\right]$. The differential of $\bar{B}_{*}(\mathcal{G})$ is given by

$$
d\left(\left[a_{1}|\cdots| a_{n}\right]\right)=\sum_{i=1}^{n-1}(-1)^{i}\left[a_{1}|\cdots| a_{i-1}\left|\mu_{\mathcal{G}}\left(a_{i} \otimes a_{i+1}\right)\right| a_{i+2}|\cdots| a_{n}\right] \quad(n>1) ;
$$

and $d\left[a_{1}\right]=0$ for all $\left[a_{1}\right] \in \bar{B}_{1}(\mathcal{G})$. The augmentation and the coaugmentation on $\bar{B}_{*}(\mathcal{G})$ coincide with the identity on $R$. Moreover, $\bar{B}_{*}(\mathcal{G})$ is a DGA-coalgebra with the coproduct:

$$
\nabla\left(\left[a_{1}|\cdots| a_{n}\right]\right)=\sum_{i=0}^{n}\left[a_{1}|\cdots| a_{i}\right] \otimes\left[a_{i+1}|\cdots| a_{n}\right] \quad(n \geqslant 0) .
$$


Let $(B, \nabla)$ be a DGA-coalgebra and $(A, \mu)$ a DGA-algebra. A twisting cochain or Brown cochain $\kappa$, is a graded module morphism $\kappa: B_{*} \rightarrow A_{*-1}$ satisfying that

$$
d_{A} \kappa+\kappa d_{B}+\mu(\kappa \otimes \kappa) \nabla=0, \quad \xi_{A} \kappa=0 \quad \text { and } \quad \kappa \eta_{B}=0 .
$$

Let $M$ be an $A$-DG-module (where $\nu: M \otimes A \rightarrow M$ is the (right) $A$-module structure on $M$ ). Define the morphism $d_{\kappa}: M \otimes B \rightarrow M \otimes B$ by

$$
d_{\kappa}(m \otimes b)=\left(d_{M \otimes B}+\kappa \cap\right)(m \otimes b)
$$

where $\kappa \cap=\left(\nu \otimes 1_{B}\right)\left(1_{M} \otimes \kappa \otimes 1_{B}\right)\left(1_{M} \otimes \nabla\right)$. The graded module $M \otimes B$ endowed with $d_{\kappa}$ is the DG-module denoted by $M \otimes_{\kappa} B$ and called the twisted tensor product by the twisting cochain $\kappa$. An example of twisted tensor product is $R_{*}[\mathcal{G}] \otimes_{\theta} \bar{B}_{*}(\mathcal{G})$, where the twisting cochain $\theta$, called the universal twisting cochain, is given by

$$
\theta\left(\left[a_{1}|\cdots| a_{n}\right]\right)=\left\{\begin{array}{cl}
a_{1} & \text { if } n=1 \\
0 & \text { otherwise. }
\end{array}\right.
$$

We deal with an special type of homotopy equivalence. A contraction $r$ from a DG-module $N$ to a DG-module $M$, consists in three morphisms $(f, g, \phi)$ where $f: N \rightarrow M$ (projection) and $g: M \rightarrow N$ (inclusion) are DG-module morphisms of degree zero and $\phi: N \rightarrow N$ (homotopy operator) increases the degree by one. Moreover, it is required that

$$
\begin{gathered}
(\mathrm{r} 1) \quad f g=1_{M}, \\
(\mathrm{r} 2) \quad \phi d+d \phi=g f-1_{N}, \\
(\mathrm{r} 3) \quad \phi g=0, f \phi=0, \phi \phi=0 .
\end{gathered}
$$

Such a contraction will be denoted by $r=(f, g, \phi): N \Rightarrow M$ or briefly $N \stackrel{r}{\Rightarrow} M$. The importance of having this structure from $M$ to $N$ is that the module $N$ has less or equal number of generators than the module $M$ although they have the same homology.

\section{Cocyclic Operations and HPT}

The goal of this section is to design an algebraic-combinatorial machinery in order to generate cocyclic operations starting from a given Eilenberg-Zilber contraction.

First of all, we recall the concept of perturbation datum and we introduce the main tool in Homological Perturbation Theory: Basic Perturbation Lemma $[\mathbf{2 3}, \mathbf{3}$, $7,8,15]$.

Let $f: M \rightarrow M$ be a DG-module morphism. The morphism $f$ is pointwise nilpotent if, for all $m \in M$, a positive integer $n(m)$ exists such that $f^{n}(m)=0$. A perturbation of a DGA-module $M$ is a graded module morphism $\varphi: M \rightarrow M$ (which decreases the degree by one), such that $\left(d_{M}+\varphi\right)^{2}=0$ and $\xi_{M} \varphi=0$. A perturbation datum of the contraction $r=(f, g, \phi): M \Rightarrow N$ is a perturbation $\varphi$ of the DGA-module $M$ satisfying that the composition $\phi \varphi$ is pointwise nilpotent.

Basic Perturbation Lemma (BPL) can be seen as a real algorithm such that the input is a contraction and a perturbation of this contraction, and the output is a new perturbed contraction. 
Theorem 3.1. Basic Perturbation Lemma [23].

INPUT:

$A$ contraction $r=(f, g, \phi):\left(M, d_{M}\right) \Rightarrow\left(N, d_{N}\right)$ and a perturbation datum $\varphi: M \rightarrow M$ of $r$.

Output:

The contraction $r_{\varphi}=\left(f_{\varphi}, g_{\varphi}, \phi_{\varphi}\right):\left(M, d_{M}+\varphi\right) \Rightarrow\left(N, d_{N}+\tilde{\varphi}\right)$ where

$$
\begin{gathered}
f_{\varphi}=\sum_{i \geqslant 0} f(\varphi \phi)^{i}, \quad g_{\varphi}=\sum_{i \geqslant 0}(\phi \varphi)^{i} g, \\
\phi_{\varphi}=-\sum_{i \geqslant 0}(\phi \varphi)^{i} \phi, \quad \tilde{\varphi}=\sum_{i \geqslant 0} f \varphi(\phi \varphi)^{i} g .
\end{gathered}
$$

Note that all the sums are finite because of the pointwise nilpotency of $\phi \varphi$.

In the following lemma, $\mathcal{G}$ is a group and $M$ and $N$ are two $R_{*}[\mathcal{G}]-$ DG-modules (where $\nu$ and $\nu^{\prime}$ are the (right) $R_{*}[\mathcal{G}]$-module structures on $M$ and $N$, respectively). If there exists a contraction $r$ form $M$ to $N$ which satisfies a commutative condition, then there exists a contraction from $M \otimes_{\theta} \bar{B}_{*}(\mathcal{G})$ to $N \otimes_{\theta} \bar{B}_{*}(\mathcal{G})$ such that the inclusion morphism is not perturbed:

\section{Lemma 3.2.}

INPUT:

A contraction $r=(f, g, \phi): M \Rightarrow N$ such that the following diagram is commutative:

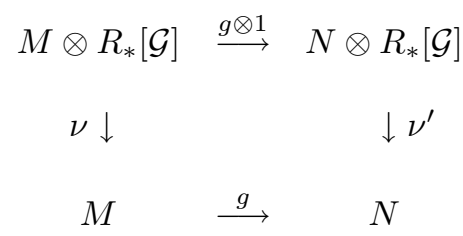

\section{Output:}

The contraction

$$
(r \otimes 1)_{\theta \cap}=\left((f \otimes 1)_{\theta \cap}, g \otimes 1,(\phi \otimes 1)_{\theta \cap}\right): M \otimes_{\theta} \bar{B}_{*}(\mathcal{G}) \Rightarrow N \otimes_{\theta} \bar{B}_{*}(\mathcal{G}) .
$$

Proof. Apply Basic Perturbation Lemma to the contraction

$$
(r \otimes 1)=(f \otimes 1, g \otimes 1, \phi \otimes 1): M \otimes \bar{B}_{*}(\mathcal{G}) \Rightarrow N \otimes \bar{B}_{*}(\mathcal{G}) .
$$

and the perturbation datum $\theta \cap$. Then, the new contraction

$$
(r \otimes 1)_{\theta \cap}=\left((f \otimes 1)_{\theta \cap},(g \otimes 1)_{\theta \cap},,(\phi \otimes 1)_{\theta \cap}\right): M \otimes_{\theta} \bar{B}_{*}(\mathcal{G}) \Rightarrow N \otimes_{\theta} \bar{B}_{*}(\mathcal{G})
$$

is obtained. Now, it is easy to see that $(g \otimes 1)(\theta \cap)=(\theta \cap)(g \otimes 1)$ due to the commutativity property (1), so that

$$
(g \otimes 1)_{\theta \cap}=g \otimes 1+\sum_{i \geqslant 1}((\phi \otimes 1)(\theta \cap))^{i}(g \otimes 1)=g \otimes 1 .
$$




\subsection{A machinery for constructing cocyclic operations}

In this subsection we design a procedure for obtained explicit formulae for cocyclic operations from the perspective of simplicial sets, which provide a combinatorial description of topological spaces. Roughly speaking, a simplicial set can be considered as an algebraic generalization of the structure of a triangulated polyhedron although the former features a more rigid combinatorial structure than the latter. We recall concepts from Simplicial Topology [19] in order to fix notation.

A simplicial set $K$ is a graded set indexed by the non-negative integers together with face and degeneracy operators $\partial_{i}: K_{q} \rightarrow K_{q-1}$ and $s_{i}: K_{q} \rightarrow K_{q+1}, 0 \leqslant i \leqslant q$, satisfying some particular commutativity properties. The elements of $K_{q}$ are called $q$-simplices. A simplex $x$ is degenerate if $x=s_{i} y$ for some simplex $y$ and degeneracy operator $s_{i}$; otherwise, $x$ is non-degenerate. Let $K$ and $L$ be two simplicial sets. The cartesian product $K \times L$ is a simplicial set whose simplices and face and degeneracy operators are given by

$$
\begin{gathered}
(K \times L)_{q}=K_{q} \times L_{q}, \\
\partial_{i}(x, y)=\left(\partial_{i} x, \partial_{i} y\right) \quad \text { and } \quad s_{i}(x, y)=\left(s_{i} x, s_{i} y\right) .
\end{gathered}
$$

The chain complex of a simplicial set $K$ with coefficients in $R$, denoted by $C_{*}(K)$, is constructed as follows: $C_{n}(K)$ is the free $R$-module on the set $K_{n}$; the face operators $\partial_{i}$ yield module maps $C_{n}(K) \rightarrow C_{n-1}(K)$, which we also call $\partial_{i}$; their alternating sum

$$
d=\sum(-1)^{i} \partial_{i}
$$

is the differential of $C_{*}(K)$. The normalized chain complex $C_{*}^{N}(K)$ is the chain complex defined as the quotient

$$
C_{n}^{N}(K)=C_{n}(K) / s\left(C_{n-1}(K)\right),
$$

where $s\left(C_{n-1}(K)\right)$ denotes the free $R$-module on the set of all the degenerate $n^{-}$ simplices of $K$. Since we will always work with normalized chain complexes, we simplify notation and write $C_{*}(K)$ instead of $C_{*}^{N}(K)$. The (co)homology of $K$ is, by definition, the (co)homology of the chain complex $C_{*}(K)$.

Eilenberg-Zilber contractions for simplicial sets $[6]$ provide the most classical example of homotopy equivalence between chain complexes in Algebraic Topology. Roughly speaking, a normalized chain complex $C_{*}(K \times L)$ reduces to the tensor product of the normalized chain complexes $C_{*}(K)$ and $C_{*}(L)$ via these contractions. A contraction of this kind is denoted by

$$
r_{E Z}=(A w, E m, S h): C_{*}(K \times L) \Rightarrow C_{*}(K) \otimes C_{*}(L) .
$$

Its morphisms have explicit formulations in terms of face and degeneracy operators. A recursive formula for the $S h$ operator is given in [5]. An explicit formula for this operator was stated by Rubio [22]. It is possible to construct a contraction from $C_{*}\left(K^{\times p}\right)$ to $C_{*}(K)^{\otimes p}$ (where $p$ is a positive integer), appropriately composing Eilenberg-Zilber contractions. If there is no confusion, it will be denoted by

$$
r_{E Z(p)}=\left(A w_{p}, E m_{p}, S h_{p}\right): C_{*}\left(K^{\times p}\right) \Rightarrow C_{*}(K)^{\otimes p} .
$$

We can now formulate our main result. 
Theorem 3.3. A Machinery for Constructing Cocyclic Operations.

INPUT:

A commutative ring $G$.

$A$ non-negative integer $p$.

$A$ subgroup $\mathcal{G}$ of the symmetric group $\mathcal{S}_{p}$.

$A$ cycle $a \in \bar{B}_{*}(\mathcal{G})$ representative of a homology class $\alpha$ in $H_{n}\left(\bar{B}_{*}(\mathcal{G}) ; G\right)$.

An Eilenberg-Zilber contraction

$$
r_{E Z(p)}=\left(A w_{p}, E m_{p}, S h_{p}\right): C_{*}\left(K^{\times p}\right) \Rightarrow C_{*}(K)^{\otimes p} .
$$

A non-negative integer $q$.

Output:

The cocyclic operation

$$
O_{\left(r_{E Z(p)}, a, q\right)}: \operatorname{Ker} \delta^{q}(K ; G) \rightarrow \operatorname{Ker} \delta^{q p-n}(K ; G / m G)
$$

(where $m=m(a, q)$ is a non-negative integer), given by

$$
O_{\left(r_{E Z(p)}, a, q\right)}(c)(x)=\mu c^{\otimes p}\left(A w_{p} \otimes 1\right)\left(\theta \cap\left(S h_{p} \otimes 1\right)\right)^{n}(\Delta x \otimes a),
$$

where $c \in \operatorname{Ker} \delta^{q}(K ; G), x \in C_{q p-n}(K), \mu$ is the product on $G / m G$, and $\Delta$ is defined by $\Delta(x)=\left(x,{ }^{p}{ }^{\text {timeses }}, x\right)$.

Proof. We have to prove that if $c \in \operatorname{Ker} \delta^{q}(K ; G)$ then $O_{\left(r_{E Z(p)}, a, q\right)}(c)$ is a $(q p-n)-$ cocycle mod $m$.

We apply Lemma 3.2 to $r_{E Z(p)}$ and the group $\mathcal{G}$, to obtain the new contraction

$$
\left(r_{E Z(p)} \otimes 1\right)_{\theta \cap}: C_{*}\left(K^{\times p}\right) \otimes_{\theta} \bar{B}_{*}(\mathcal{G}) \Rightarrow C_{*}(K)^{\otimes p} \otimes_{\theta} \bar{B}_{*}(\mathcal{G}) .
$$

Now, since $\left(A w_{p} \otimes 1\right)_{\theta \cap}$ is a DG-module morphism, we have that

$$
\begin{aligned}
& \left(A w_{p} \otimes 1\right)_{\theta \cap}(1 \otimes d+d \otimes 1+\theta \cap)(\Delta x \otimes a) \\
& =(1 \otimes d+d \otimes 1+\theta \cap)\left(A w_{p} \otimes 1\right)_{\theta \cap}(\Delta x \otimes a) .
\end{aligned}
$$

for all $x \in C_{p q-n}(K)$. On one hand, since $d(a)=0$ then

$$
\left(A w_{p} \otimes 1\right)_{\theta \cap}(1 \otimes d)(\Delta x \otimes a)=0 .
$$

On the other hand, since the symmetric group $\mathcal{S}_{p}$ operates on $C_{*}\left(K^{\times p}\right)$ by the usual action, then $\theta \cap(\Delta x \otimes a)=0$, so

$$
\left(A w_{p} \otimes 1\right)_{\theta \cap} \theta \cap(\Delta x \otimes a)=0 .
$$

Now, (2) becomes

$$
\begin{aligned}
& \left(A w_{p} \otimes 1\right)_{\theta \cap}(d \otimes 1)(\Delta x \otimes a) \\
& =(1 \otimes d+d \otimes 1+\theta \cap)\left(A w_{p} \otimes 1\right)_{\theta \cap}(\Delta x \otimes a) .
\end{aligned}
$$

Adding $\mu c^{\otimes p}\left(1 \otimes \xi_{\bar{B}[\mathcal{G}]}\right)$ to the both sides of (3), we have

$$
\begin{aligned}
& \mu c^{\otimes p}\left(1 \otimes \xi_{\bar{B}[\mathcal{G}]}\right)\left(A w_{p} \otimes 1\right)_{\theta \cap}(d \otimes 1)(\Delta x \otimes a) \\
& =\mu c^{\otimes p}\left(1 \otimes \xi_{\bar{B}[\mathcal{G}]}\right)(1 \otimes d+d \otimes 1+\theta \cap)\left(A w_{p} \otimes 1\right)_{\theta \cap}(\Delta x \otimes a) .
\end{aligned}
$$

Since $d\left[a_{1}\right]=0$ for any $\left[a_{1}\right] \in \bar{B}_{1}(\mathcal{G})$ then

$$
\mu c^{\otimes p}\left(1 \otimes \xi_{\bar{B}[\mathcal{G}]}\right)(1 \otimes d)\left(A w_{p} \otimes 1\right)_{\theta \cap}(\Delta x \otimes a)=0 .
$$


Since $c$ is a cocycle then

$$
\mu c^{\otimes p}\left(1 \otimes \xi_{\bar{B}[\mathcal{G}]}\right)(d \otimes 1)\left(A w_{p} \otimes 1\right)_{\theta \cap}(\Delta x \otimes a)=0 .
$$

Taking into account that the symmetric group $\mathcal{S}_{p}$ operates on $C_{*}(K)^{\otimes p}$ by the usual action, then

$$
\mu c^{\otimes p}\left(1 \otimes \xi_{\bar{B}[\mathcal{G}]}\right) \theta \cap\left(A w_{p} \otimes 1\right)_{\theta \cap}(\Delta x \otimes a)=0 \quad \bmod m,
$$

where $m$ is a non-negative integer depending on the cycle $a$ and the integer $q$. Substituting these results in (4), we have that

$$
\mu c^{\otimes p}\left(1 \otimes \xi_{\bar{B}[\mathcal{G}]}\right)\left(A w_{p} \otimes 1\right)_{\theta \cap}(d \otimes 1)(\Delta x \otimes a)=0 \quad \bmod m,
$$

Then,

$$
\mu c^{\otimes p}\left(A w_{p} \otimes 1\right)\left(\theta \cap\left(S h_{p} \otimes 1\right)\right)^{n}(\Delta d x \otimes a)=0 \quad \bmod m,
$$

and finally we get

$$
\delta O_{\left(r_{E Z(p)}, a, q\right)}(c)(x)=0 \quad \bmod m .
$$

That is, $O_{\left(r_{E Z(p)}, a, q\right)}(c)$ is a $(p q-n)$-cocycle $\bmod m$ for any $q$-cocycle $c$.

We analyze the case of cocyclic operations constructed with the machinery using different cycles (representative of the same homology class) or different EilenbergZilber contractions in [13].

\section{Examples}

In this section, we give two examples of the application of the technique explained before. The first one is a reinterpretation of the work of Steenrod concerning to Steenrod squares and Steenrod powers operations $[\mathbf{2 4}, \mathbf{2 5}]$. The second one is concerning to the computation of Adem secondary cocyclic operations $[\mathbf{1}, \mathbf{2}]$.

\subsection{Steenrod Cocyclic Operations}

In this subsection, we give a new proof of Theorem 3.1 of [9] about explicit expressions of Steenrod cocyclic operations as an example of the application of the method explained above.

First, let us recall the definition of these particular operations. An infinite sequence of morphisms $\left\{D_{n}\right\}_{n \geqslant 0}$ which "measures" the lack of commutativity of $A w$ is called a higher diagonal approximation [25]. More concretely, given a simplicial set $K$, and an Eilenberg-Zilber contraction $r_{E Z(p)}=\left(A w_{p}, E m_{p}, S h_{p}\right)$, a higher diagonal approximation is a sequence of morphisms $D_{n}: C_{*}(K) \rightarrow C_{*}(K)^{\otimes p}$ of degree $n$ such that:

$$
D_{0}=A w_{p} \Delta, \quad d_{C_{*}(K) \otimes p} D_{n}+(-1)^{n-1} D_{n} d_{C_{*}(K)}=\gamma_{n}^{\prime} D_{n-1} ;
$$

where $\gamma_{n}^{\prime}: C_{*}(K)^{\otimes p} \rightarrow C_{*}(K)^{\otimes p}$ is defined by

$$
\gamma_{n}^{\prime}= \begin{cases}T-1 & \text { if } n \text { odd } \\ 1+T+\cdots+T^{p-1} & \text { if } n \text { even }\end{cases}
$$


and $T: C_{*}(K)^{\otimes p} \rightarrow C_{*}(K)^{\otimes p}$ is the cyclic permutation defined by

$$
T\left(x_{1} \otimes x_{2} \otimes \cdots \otimes x_{p}\right)=(-1)^{\left|x_{1}\right|\left(\left|x_{2}\right|+\cdots+\left|x_{p}\right|\right)} x_{2} \otimes \cdots \otimes x_{p} \otimes x_{1} .
$$

Given a higher diagonal approximation $\left\{D_{n}\right\}$, Steenrod cocyclic operations [25] are defined by:

$$
\mathcal{P}_{n}^{p}(c)=\mu c^{\otimes p} D_{n} \in \operatorname{Ker} \delta^{q p-n}\left(K ; \mathbf{Z}_{p}\right),
$$

where $p$ is a prime number, $\mu$ is the natural product on $\mathbf{Z}_{p}$ and $c \in \operatorname{Ker} \delta^{q}(K ; \mathbf{Z})$.

The acyclic model method [4] is used for guaranteeing the existence of the morphisms $D_{n}$. Nevertheless, working in a simplicial framework, another constructive approximation to these morphisms can be made. In order to obtain this, we apply Theorem 3.3 to the cyclic group $\mathbf{Z}_{p}$ and the family of cycles

$$
\left\{a_{n}=\sum_{\bar{x}_{i} \in \mathbf{Z}_{p}}\left[\overline{1}-\overline{0}\left|\bar{x}_{1}-\overline{0}\right| \overline{1}-\overline{0}\left|\bar{x}_{2}-\overline{0}\right| \cdots\right] \in \bar{B}_{n}\left(\mathbf{Z}_{p}\right)\right\}_{n \geqslant 0} .
$$

The $\mathbf{Z}\left[\mathbf{Z}_{p}\right]-$ module structures on $C_{*}\left(K^{\times p}\right)$ and $C_{*}(K)^{\otimes p}$ are, respectively:

$$
\nu(x, i)=t^{i}(x) \quad \text { and } \quad \nu^{\prime}(y, i)=T^{i}(y) \quad\left(i \in \mathbf{Z}_{p}\right),
$$

where $x \in C_{*}\left(K^{\times p}\right), y \in C_{*}(K)^{\otimes p}$, $t$ is the cyclic permutation on $C_{*}\left(K^{\times p}\right)$ given by $t\left(x_{1}, x_{2}, \ldots, x_{p}\right)=\left(x_{2}, \ldots, x_{p}, x_{1}\right)$, and $T$ is that on $C_{*}(K)^{\otimes p}$.

The output of Theorem 3.3 gives the family of cocyclic operations

$$
\left\{O_{\left(r_{E Z(p)}, a_{n}, q\right)}: \operatorname{Ker} \delta^{q}(K ; \mathbf{Z}) \rightarrow \operatorname{Ker} \delta^{q p-n}\left(K ; \mathbf{Z}_{p}\right)\right\}_{n, q \geqslant 0},
$$

such that, if $c \in \operatorname{Ker} \delta^{q}(K ; \mathbf{Z})$ and $x \in C_{q p-n}(K)$ then

$$
\begin{aligned}
O_{\left(r_{E Z(p)}, a_{n}, q\right)}(c)(x) & =\mu c^{\otimes p}\left(A w_{p} \otimes 1\right)\left(\theta \cap\left(S h_{p} \otimes 1\right)\right)^{n}\left(\Delta x \otimes a_{n}\right) \\
& =\sum_{\bar{x}_{i} \in \mathbf{Z}_{p}} \mu c^{\otimes p} A w_{p}\left(\cdots \nu\left(S h_{p} \nu\left(S h_{p} \Delta x \otimes(\overline{1}-\overline{0}) \otimes\left(x_{1}-\overline{0}\right)\right) \cdots\right)\right. \\
& =\mu c^{\otimes p} A w_{p} \gamma_{n} S h_{p} \cdots \gamma_{1} S h_{p} \Delta x
\end{aligned}
$$

where, for all $1 \leqslant j \leqslant n, \gamma_{j}: C_{*}\left(K^{\times p}\right) \rightarrow C_{*}\left(K^{\times p}\right)$ is defined by

$$
\gamma_{j}=\left\{\begin{array}{cl}
t & \text { if } j \text { odd } \\
t+\cdots+t^{p-1} & \text { if } j \text { even. }
\end{array}\right.
$$

\subsection{Adem Secondary Cocyclic Operations}

In $[\mathbf{1}, \mathbf{2}]$, J. Adem constructed secondary cohomology operations using relations on iterated Steenrod squares. He proved the relation $S q^{2} S q^{2}+S q^{3} S q^{1}=0$ by means of a cochain construction. More precisely, he established the existence of cochain mappings

$$
\mathcal{E}_{j}: C^{p}\left(K^{\times 4} ; \mathbf{Z}\right) \rightarrow C^{4 p-j}\left(K ; \mathbf{Z}_{2}\right)
$$

such that $\bmod 2$

$$
\left(c \smile_{i} c\right) \smile_{i+2}\left(c \smile_{i} c\right)+\left(c \smile_{i+1} c\right) \smile_{i}\left(c \smile_{i+1} c\right)=\delta \mathcal{E}_{3 i+3}\left(c^{4}\right)
$$


where $\smile_{k}$ is the cup- $k$ product [24], $c$ is a $q-$ cocycle and $i=q-2$. He demonstrated that if $c$ is a $q$-cocycle such that $S q^{2}(c)$ is a coboundary (that is, there exists a cochain $b$ such that $c \smile_{i} c=\delta b$ ), then

$$
w=b \smile_{i+1} b+b \smile_{i+2} \delta b+\mathcal{E}_{3 i+3}\left(c^{4}\right)+\eta(c) \smile_{i-1} \eta(c)+\eta(c) \smile_{i} \delta \eta(c)
$$

is a mod 2 cocycle, where $\eta(c)=\frac{1}{2}\left(c \smile_{i+2} c\right)+c$. Therefore, Adem secondary cohomology operations are defined as

$$
\Psi_{q}[c]=[w]+S q^{2} H^{q+1}(K ; \mathbf{Z}) \in H^{q+3}\left(K ; \mathbf{Z}_{2}\right) .
$$

Now, we explain our procedure to obtain explicit formulas for the operation $\Psi_{2}[c]$ at cocycle level. An study of the general case $\Psi_{q}[c]$ is done in [14].

Our aim is to obtain an explicit formula for $\mathcal{E}_{3}$. Consider the semi-direct product $\mathcal{G}=\mathbf{Z}_{2}^{\times 2} \times_{\chi} \mathbf{Z}_{2}$ where

$$
\chi(\bar{a}, \bar{b}, \overline{1})=(\bar{b}, \bar{a}) .
$$

The $\mathbf{Z}_{2}[\mathcal{G}]$-module structures on $C_{*}\left(K^{\times 4}\right)$ and $C_{*}(K)^{\otimes 4}$ are given by:

$$
\begin{gathered}
\nu\left(x, e_{1}\right)=z(x)=\left(x_{1}, x_{3}, x_{2}, x_{4}\right), \\
\nu\left(x, e_{2}\right)=(t \times t)(x), \quad \nu\left(x, e_{3}\right)=t(x)
\end{gathered}
$$

and

$$
\begin{gathered}
\nu^{\prime}\left(y, e_{1}\right)=z^{\prime}(y)=(-1)^{\left|y_{2}\right| \cdot\left|y_{3}\right|} y_{1} \otimes y_{3} \otimes y_{2} \otimes y_{4}, \\
\nu^{\prime}\left(y, e_{2}\right)=(T \otimes T)(y), \quad \nu^{\prime}\left(y, e_{3}\right)=T(y)
\end{gathered}
$$

where $x=\left(x_{1}, x_{2}, x_{3}, x_{4}\right) \in C_{*}\left(K^{\times 4}\right), \quad y=y_{1} \otimes y_{2} \otimes y_{3} \otimes y_{4} \in C_{*}(K)^{\otimes 4}$ and

$$
e_{1}=(\overline{0}, \overline{0}, \overline{1}), \quad e_{2}=(\overline{1}, \overline{0}, \overline{0}), \quad e_{3}=(\overline{0}, \overline{1}, \overline{0}) .
$$

Consider the Eilenberg-Zilber contraction

$$
r_{E Z(4)}=\left(A w_{4}, E m_{4}, S h_{4}\right): C_{*}\left(K^{\times 4}\right) \stackrel{r_{E Z}}{\Rightarrow}\left(C_{*}\left(K^{\times 2}\right)\right)^{\otimes 2} \stackrel{r_{E Z} \stackrel{\otimes}{r_{E Z}}}{\Rightarrow} C_{*}(K)^{\otimes 4} .
$$

Let us take the following boundary in $\bar{B}_{3}\left(\mathbf{Z}_{2}^{\times 2} \times{ }_{\chi} \mathbf{Z}_{2}\right)$ :

$$
\begin{aligned}
a_{3}= & {\left[e_{3}-e_{0}\left|e_{3}-e_{0}\right| e_{1}-e_{0}\right] } \\
& +\left[e_{3}-e_{0}\left|e_{1}-e_{0}\right| e_{2}-e_{0}\right]+\left[e_{1}-e_{0}\left|e_{2}-e_{0}\right| e_{2}-e_{0}\right] \quad \bmod 2
\end{aligned}
$$

where $e_{0}=(\overline{0}, \overline{0}, \overline{0})$. It satisfies that

$$
d\left(a_{3}\right)=\left[\left(e_{3}-e_{0} \mid e_{3}-e_{0}\right]+\left[e_{2}-e_{0} \mid e_{2}-e_{0}\right]=b_{2} \quad \bmod 2 .\right.
$$

Since $a_{3}$ is not a cycle, we can not directly apply Theorem 3.3. But, following an analog process, we apply Lemma 3.2 to the contraction $r_{E Z(4)}$ and the group $\mathcal{G}$, to obtain the new contraction

$$
\left(r_{E Z(4)} \otimes 1\right)_{\theta \cap}: C_{*}\left(K^{\times 4}\right) \otimes_{\theta} \bar{B}_{*}(\mathcal{G}) \Rightarrow C_{*}(K)^{\otimes 4} \otimes_{\theta} \bar{B}_{*}(\mathcal{G}) .
$$

Now, since $\left(A w_{4} \otimes 1\right)_{\theta \cap}$ is a DG-module morphism, we have that mod 2,

$$
\begin{aligned}
& \left(A w_{4} \otimes 1\right)_{\theta \cap}(1 \otimes d+d \otimes 1+\theta \cap)\left(\Delta x \otimes a_{3}\right) \\
& =(1 \otimes d+d \otimes 1+\theta \cap)\left(A w_{4} \otimes 1\right)_{\theta \cap}\left(\Delta x \otimes a_{3}\right)
\end{aligned}
$$


Simplifying this equation and adding $\mu c^{\otimes 4}\left(1 \otimes \xi_{\bar{B}[\mathcal{G}]}\right)$ to the both sides of (7) we have that $\bmod 2$ :

$$
\begin{aligned}
& \mu c^{4}\left(1 \otimes \xi_{\bar{B}[\mathcal{G}]}\right)\left(A w_{4} \otimes 1\right)_{\theta \cap}\left(\Delta x \otimes b_{2}\right) \\
& =\mu c^{4}\left(1 \otimes \xi_{\bar{B}[\mathcal{G}]}\right)\left(A w_{4} \otimes 1\right)_{\theta \cap}\left(\Delta d x \otimes a_{3}\right)
\end{aligned}
$$

for all $c \in \operatorname{Ker} \delta^{2}(K ; \mathbf{Z})$ and $x \in C_{6}(K)$. On one hand, simplifying the right term of $(8)$, we obtain the following identities $\bmod 2$ :

$$
\begin{aligned}
& \mu c^{4}\left(1 \otimes \xi_{\bar{B}[\mathcal{G}]}\right)\left(A w_{4} \otimes 1\right)\left(\theta \cap\left(S h_{4} \otimes 1\right)\right)^{3}\left(\Delta x \otimes b_{2}\right) \\
& =\mu c^{4}\left(A w_{4} \otimes 1\right)\left(\theta \cap\left(S h_{4} \otimes 1\right)\right)^{2}\left(\Delta x \otimes b_{2}\right) \\
& =\mu c^{4}\left(A w_{4}(t \times t) S h_{4}(t \times t) S h_{4} z \Delta(x)+A w_{4} t S h_{4} t S h_{4} \Delta(x)\right) \\
& =\left(c \smile_{2} c\right) \smile_{0}\left(c \smile_{0} c\right)+\left(c \smile_{1} c\right) \smile_{0}\left(c \smile_{1} c\right)+\left(c \smile_{0} c\right) \smile_{0}\left(c \smile_{2} c\right) \\
& +\left(c \smile_{0} c\right) \smile_{2}\left(c \smile_{0} c\right) \\
& =\left(c \smile_{2} c\right) \smile_{0}\left(c \smile_{0} c\right)+\left(c \smile_{1} c\right) \smile_{0}\left(c \smile_{1} c\right)+\delta\left(\left(c \smile_{0} c\right) \smile_{1}\left(c \smile_{2} c\right)\right) .
\end{aligned}
$$

On the other hand, the left term is:

$$
\begin{aligned}
& \mu c^{p}\left(1 \otimes \xi_{\bar{B}[\mathcal{G}]}\right)\left(A w_{4} \otimes 1\right)_{\theta \cap}\left(\Delta d x \otimes a_{3}\right) \\
& =\mu c^{4}\left(A w_{4} \otimes 1\right)\left(\theta \cap\left(S h_{4} \otimes 1\right)\right)^{3}\left(\Delta d x \otimes a_{3}\right) \\
& =\mu c^{4}\left(A w_{4}(t \times t) S h_{4}(t \times t) S h_{4} z S h_{4} \Delta d(x)+A w_{4}(t \times t) S h_{4} z S h_{4} t S h_{4} \Delta d(x)\right. \\
& \left.\quad+A w_{4} z S h_{4} t S h_{4} t S h_{4} \Delta d(x)\right) \\
& =\mu c^{4}\left(\left(D_{2} \otimes A w+D_{1} t \otimes T D_{1}\right) A w z(S h+E m(S h \otimes E m A w+1 \otimes S h) A w)\right. \\
& \quad+\left(D_{1} \otimes T A w+A w t \otimes D_{1}\right) A w z(S h t S h+E m(S h \otimes S h) A w) \\
& \quad+(A w \otimes A w) A w z S h t S h t S h) \Delta d(x),
\end{aligned}
$$

where $D_{i}=A w(t S h)^{i}$. Then, defining

$$
\begin{aligned}
\mathcal{E}_{3}=\mu c^{4} & \left(D_{2} \otimes A w+D_{1} t \otimes T D_{1}\right) A w z(S h+\operatorname{Em}(\operatorname{Sh} \otimes \operatorname{Em} A w+1 \otimes S h) A w) \\
& +\left(D_{1} \otimes T A w+A w t \otimes D_{1}\right) A w z(\operatorname{ShtSh}+\operatorname{Em}(\operatorname{Sh} \otimes S h) A w) \\
& +(A w \otimes A w) A w z S h t S h t S h) \Delta \\
+(c & \left.\smile_{0} c\right) \smile_{1}\left(c \smile_{2} c\right),
\end{aligned}
$$

the relation (8) is simplified to:

$$
\delta\left(\mathcal{E}_{3}\right)=\left(c \smile_{2} c\right) \smile_{0}\left(c \smile_{0} c\right)+\left(c \smile_{1} c\right) \smile_{0}\left(c \smile_{1} c\right) .
$$

So, we have obtained the explicit morphism $\mathcal{E}_{j}$ in the case $j=3$, which is used to construct the Adem secondary operation $\Psi_{2}$.

\section{Comments}

In this paper, we give a method for obtaining explicit formulae for cocyclic operations in terms of the explicit morphisms of a given Eilenberg-Zilber contraction. Since shuffles (a special type of permutation) of degeneracy operators are involved in the formulae of cocyclic operations, an algorithm designed for computing the operations from these formulae would be too slow for practical implementation. Because of this, the idea of simplification arises in a natural way. This normalization 
process is based on the fact that any composition of face and degeneracy operators can be uniquely expressed in terms of face operators. Some work have been done in this way by the authors (see $[\mathbf{1 2}]$ ).

Cohomology operations that can be explicitly expressed at cochain level, can be computed via an explicit contraction from the (co)chain complex of $K$ to a "minimal" (co)chain complex $M(K)$ (see $[\mathbf{1 0}]$ ), using the well-known reduction algorithm for computing homology $[\mathbf{2 1}]$. This contraction satisfies that whenever the ground ring is a field $F$ or the (co)homology of $K$ is free, then $M(K)$ is isomorphic to the (co)homology of $K$. Therefore, an algorithm for computing the cohomology operation

$$
\mathcal{O}_{(q, r, F)}: H^{q}(K, F) \rightarrow H^{r}(K ; F)
$$

can be designed as follows:

INPUT:

A simplicial set $K$ finite in each degree.

A contraction $\left(f^{*}, g^{*}, \phi^{*}\right)$ from $C^{*}(K ; F)$ to $H^{*}(K ; F)$.

An explicit expression, in terms of face operators of $K$, of the cocyclic operation

$$
O_{(q, r, F)}: C^{q}(K, F) \rightarrow C^{r}(K ; F)
$$

such that $\mathcal{O}(\alpha)=[O(a)]$ for any $\alpha \in H^{q}(K, F)$ and $a \in C^{q}(K, F)$ such that $[a]=\alpha$.

An element $\alpha$ in $H^{q}(K ; F)$.

\section{Output:}

The element $\mathcal{O}_{(q, r, F)}(\alpha)=f^{*}\left(O_{(q, r, F)}\left(g^{*}(\alpha)\right)\right)$ in $H^{r}(K, F)$.

Note that the complexity of this algorithm essentially depends on the complexity of the explicit expressions of the cohomology operation $\mathcal{O}$ at cocyclic level.

Using all these results, [10] is devoted to design and practical implement an algorithm for computing the first Adem cohomology operation $\Psi_{2}$. Moreover, in [14], the computation of all Adem cohomology operations is studied in detail.

Nevertheless, a lot of work is needed to be done in order to obtain a general scheme for computing all the cohomology operations using this approach. We need to study the hypothesis under which Theorem 3.3 provides cocyclic operations that are also coboundary operations (that is, that preserve coboundaries). In this way, we will obtain a real cohomology operation at cochain level. Moreover, it is interesting the study of conditions under which cocyclic operations are homomorphisms.

\section{References}

[1] J. Adem. The iteration of the Steenrod Squares in Algebraic Topology. Proc. Nat. Acad. Sci. USA, vol. 38 (1952) 720-724.

[2] J. Adem. Operaciones Cohomológicas de Segundo Orden Asociadas a Cuadrados de Steenrod. Symposium Internacional de Topología Algebraica, Univ. of Mexico, Mexico D.F. (1958) 186-221. 
[3] R. Brown. The Twisted Eilenberg-Zilber Theorem. Celebrazioni Archimedee del secolo XX, Simposio di topologia (Messina, 1964), Ed. Oderisi, Gubbio (1965), 33-37.

[4] S. Eilenberg, S. McLane. Acyclic Models. Am. J. Math. vol. 67 (1953), 282 312 .

[5] S. Eilenberg, S. McLane. On the groups $H(\pi, n)$, II. Ann. of Math. vol. 60 (1954), 49-139.

[6] S. Eilenberg, J.A. Zilber. On Products of Complexes. Am. J. Math. vol. 75 (1959), 200-204.

[7] V.K.A.M. Gugenheim, L. Lambe. Perturbation Theory in Differential Homological Algebra, I. Illinois J. Math vol. 33 (1989), 56-582.

[8] V.K.A.M. Gugenheim, L. Lambe, J. Stasheff. Perturbation Theory in Differential Homological Algebra, II. Illinois J. Math. vol. 35 (3) (1991), 357-373.

[9] R. González-Díaz, P. Real. A Combinatorial Method for Computing Steenrod Squares. J. of Pure and Applied Algebra vol. 139 (1999), 89-108.

[10] R. González-Díaz, P. Real. Geometric Objects and Cohomology Operations. Proceedings of Computer Algebra and Scientific Computing (2002), 121-130.

[11] R. González-Díaz, P. Real. Computation of Cohomology Operations on Finite Simplicial Complexes. Homology, Homotopy and Applications, vol. 5 (2) (2003), 83-93.

[12] R. González-Díaz, P. Real. Simplification Techniques for Maps in Simplicial Topology. Article submitted to Journal of Simbolic Computation.

[13] R. González-Díaz, P. Real. Cohomology Operations and Homological Perturbation Theory. Preprint.

[14] R. González-Díaz, P. Real. Computing Adem Secondary Cohomology Operations. Preprint.

[15] J. Huebschmann, T. Kadeishvili. Small Models for Chain Algebras. Math. Z. vol. 207 (1991), 245-280.

[16] S. Klaus. Cochain Operations and Higher Cohomology Operations. Cahiers de Topologie et Geometrie Differentielles Categoriques.

[17] L. Kristensen. On Secondary Cohomology Operations. Math. Scand. 12 (1963), 57-82.

[18] L. Kristensen, I. Madsen. On Evaluation of Higher Order Cohomology Operations. Math. Scand. 20, 114-130 (1967).

[19] P. May. Simplicial Objects in Algebraic Topology. Chicago Lecture in Math., the Univ. of Chicago Press, 1967.

[20] S. McLane. Homology. Classics in Math., Springer-Verlag, Berlin, 1995.

[21] J.R. Munkres. Elements of Algebraic Topology. Addison-Wesley Co., 1984.

[22] J. Rubio. Homologie effective des espaces de lacets itérés: un logiciel. Thèse de doctorat de l'Institut Fourier, Grenoble (1991).

[23] W. Shih. Homologie des Espaces Fibrés. Inst. Hautes Etudes Sci. vol.13 (1962), 93-176. 
[24] N.E. Steenrod. Products of Cocycles and Extensions of Mappings. Ann. of Math. vol. 48 (1947), 290-320.

[25] N.E. Steenrod. Reduced Powers of Cohomology Classes. Ann. of Math. vol. 56 (1952), 47-67.

This article may be accessed via WWW at http://www.rmi.acnet.ge/hha/ or by anonymous ftp at

ftp://ftp.rmi.acnet.ge/pub/hha/volumes/2005/n2a5/v7n2a5.(dvi,ps,pdf)

Rocio Gonzalez-Diaz rogodi@us.es

Applied Maths Department

University of Seville

Spain

Seville

Avda. Reina Mercedes, s/n

Pedro Real real@us.es

Applied Maths Department

University of Seville

Spain

Seville

Avda. Reina Mercedes, s/n 